\title{
NECESSARY CONDITIONS FOR STABILITY OF NONSINGULAR ENDOMORPHISMS OF THE CIRCLE
}

\author{
CARLOS ARTEAGA
}

\begin{abstract}
In this article we prove that Axiom $\mathbf{A}$ is a necessary condition for structural stability of $C^{1}$ nonsingular endomorphisms of the circle.
\end{abstract}

1. Introduction. We say that an endomorphism $f: S^{1} \rightarrow S^{1}$ of the circle is nonsingular if $d f$ is injective at each point of $S^{1}$. Let $N\left(S^{1}\right)$ be the space of $C^{1}$ nonsingular endomorphisms of $S^{1}$ endowed with the $C^{1}$ topology. $f$ in $N\left(S^{1}\right)$ is said to be structurally stable if it has a neighborhood $\mathcal{U}$ such that any $g \in \mathcal{U}$ is topologically conjugate to $f$; i.e., there exists a homeomorphism $h: S^{\mathbf{1}} \rightarrow S^{\mathbf{1}}$ satisfying $h f=g h$. In [4] Z. Nitecki proves that Axiom A is a sufficient condition for a nonsingular endomorphism of $S^{1}$ to be structurally stable. Recall that $f \in N\left(S^{1}\right)$ satisfies Axiom A if

(a) $\overline{\operatorname{Per}(f)}=\Omega(f)$. Here $\operatorname{Per}(f)$ denotes the set of all periodic points of $f$ and $\Omega(f)$ the set of nonwandering points of $f$, i.e., $x \in \Omega(f)$ if and only if for any neighborhood $U$ of $x$ there is an integer $n>0$ such that $f^{n}(U) \cap U \neq \emptyset$.

(b) $\Omega(f)$ has a hyperbolic structure, i.e., $\Omega(f)$ decomposes into a disjoint union of two closed, invariant subsets $\Omega(f)=\Omega_{c} \cup \Omega_{e}$ such that there exist $k>0,0<\lambda<1$, satisfying

$$
\left|d f^{n}(x)\right| \leq k \lambda^{n} \quad \text { for all } x \in \Omega_{c}
$$

and

$$
\left|d f^{n}(x)\right| \geq k \lambda^{-n} \text { for all } x \in \Omega_{e} .
$$

The purpose of this paper is to prove that the condition above is necessary for structural stability.

THEOREM. If $f \in N\left(S^{1}\right)$ is structurally stable then $f$ satisfies Axiom A.

A fundamental tool for the proof of the theorem will be a lemma (Lemma 3.1) essentially contained in the proof of a theorem of Jakobson [2, Theorem A].

The author thanks R. Mañe for helpful discussions about this paper.

2. Preliminaries. Let $f \in N\left(S^{1}\right)$. A periodic point $x$ of periodic $n$ is called hyperbolic, contracting or expanding according as $\left|d f^{n}(x)\right| \neq 1,<1$ or $>1$, respectively. Let $\Omega_{c}(f)$ denote the set of contracting periodic points of $f$. The set $\Omega(f)-\Omega_{c}(f)$ will be denoted by $\Omega_{e}(f)$.

Let $x \in \Omega_{c}(f)$. The stable manifold of $x, W^{\mathrm{s}}(x)$, is defined by $W^{\mathbf{s}}(x)=\{y: x \in$ $\omega(y)\}$. Here $\omega(y)$ denotes the $\omega$-limit set of the orbit $\left\{f^{n}(y)\right\}$. In general, $W^{\mathbf{s}}(x)$ consists of countably many disjoint intervals. The interval containing $x$ will be called the local stable manifold and is denoted by $W_{l}^{\mathrm{s}}(x)$. The stable manifold of $f$,

Received by the editors May 2, 1983, and, in revised form, July 11, 1983.

1980 Mathematics Subject Classification. Primary 34C35; Secondary 34D30.

(C) 1984 American Mathematical Society $0002-9939 / 84 \$ 1.00+\$ .25$ per page 
$\Delta(f)$, is defined by $\Delta(f)=\bigcup W^{\mathrm{s}}(x)$, where the union is taken over all contracting periodic points $x$ of $f$. We let $\Sigma(f)=S^{1}-\Delta(f)$.

A point $x$ is eventually periodic if some iterate of $x$ is a periodic point of $f$. A point $x$ is called recurrent if $x \in \omega(x)$.

3. Proof of the theorem. We start establishing some preliminary results.

The following lemma is a consequence of Theorem 3 and the Remark to Theorem 3 of $[2]$.

LEMMA 3.1. Let $f$ be a $C^{2}$ nonsingular endomorphism of $S^{1}$ satisfying:

(i) All periodic points are hyperbolic;

(ii) $f$ has a finite number (nonzero) of contracting periodic points.

Then $\Sigma(f)$ is totally disconnected, and $\left.f\right|_{\Sigma(f)}$ and $\left.f\right|_{\Omega_{e}(f)}$ are topologically conjugate to subsemishifts of finite type.

Using this result it is possible to prove the following lemmas.

LEMMA 3.2. Let $f \in N\left(S^{1}\right)$ be structurally stable of degree $>1$, and let $\varepsilon>0$. If $p \in \Omega_{e}(f)$ is a recurrent point of $f$, then there exists $q \in \operatorname{Per}(f)$ such that $\left|f^{j}(p)-f^{j}(q)\right|<\varepsilon$ for all $0 \leq j<m$, where $m$ is the period of $p$.

PROOF. We divide the proof of the lemma in two cases.

(1) $\Omega_{c}(f) \neq \emptyset$. By hypothesis $f$ has all periodic points hyperbolic.

Choose a $C^{2}$ nonsingular endomorphism $f_{1}$ sufficiently close to $f$ in the $C^{1}$ topology. By hypothesis $f_{1}$ and $f$ are topologically conjugate. It follows from this that $f_{1}$ has all periodic points hyperbolic and $\Omega_{c}\left(f_{1}\right) \neq \emptyset$, because $f$ has these properties and a conjugacy preserves hyperbolic periodic points and nonwandering points. By Szlenk's Lemma [2, Lemma 4], $f_{1}$ can be approximated in the $C^{1}$ topology by a $C^{2}$ nonsingular endomorphism $f_{2}$ satisfying the hypothesis of Lemma 3.1. Hence, $f_{2 \mid \Omega_{e}\left(f_{2}\right)}$ is topologically conjugate to a subsemishift of finite type, and since $f_{2}$ and $f$ are topologically conjugate, $\left.f\right|_{\Omega_{e}(f)}$ is also topologically conjugate to a subsemishift of finite type. Therefore, there is a periodic point $q \in \Omega_{e}(f)$ such that $\left|f^{j}(p)-f^{j}(q)\right|<\varepsilon$ for all $0 \leq j \leq m$, where $m$ is the period of $q$.

(2) $\Omega_{c}(f)=\emptyset$. By $[2$, Lemma 5$], \Omega(f)=S^{1}$. Hence by [5, Lemma 3.1], $f$ is topologically conjugate to the expanding map $\delta_{d}: S^{1} \rightarrow S^{1}$ defined by $\delta_{d}(z)=z^{d}$, where $d=\operatorname{degree} f$. Then the lemma follows from the fact that $\delta_{d}$ satisfies the property of the lemma and the uniform continuity of the conjugation.

LEMMA 3.3. Let $f \in N\left(S^{1}\right)$ be structurally stable of degree $>1$. If $p \in \Omega_{e}(f)$ is a recurrent point of $f$ then there exists $n \in N$ such that $\left|d f^{n}(p)\right|>1$.

ProOF. By the proof of Lemma 3.2, $\Omega_{c}(f)$ is finite.

Choose $0<\varepsilon<1$ and a compact neighborhood $U_{c}$ of $\Omega_{c}(f)$ such that if $g$ is $\varepsilon$ close to $f$ in the $C^{1}$ metric then $g$ is topologically conjugate to $f$ and $\Omega_{c}(g) \subset U_{c}$. By Lemma 3.2 and the fact that $d f$ is uniformly continuous, there exists a periodic point $q \in \Omega_{e}(f)$ such that $\left|d f\left(f^{l}(p)\right)-d f\left(f^{l}(q)\right)\right|<\varepsilon / 3$ for all $0 \leq l<n$, where $n$ is the period of $q$.

Now, to prove the lemma we shall adapt techniques due to Franks [1] and Mañe [3]. Choose a number $\delta, 0<\delta<\varepsilon / 3$, such that if $I_{l}=\left\{x \in S^{1}|| x-f^{l}(q) \mid<\delta\right\}$ then $I_{l} \cap U_{c}=\emptyset$ for all $0 \leq l<n$, and $I_{i}$ and $I_{j}$ are disjoint when $i \neq j$. 
For every $l=0, \ldots, n-1$, choose a $C^{\infty}$ real valued function $\sigma_{l}$ such that $0 \leq$ $\sigma_{l}(x) \leq 1, \sigma_{l}(x)=0$ if $x \in S^{1}-I_{l}, \sigma_{l}(x)=1$ if $\left|x-f^{l}(q)\right| \leq \delta / 4$ and $\left|\sigma_{l}^{\prime}(x)\right|<2 / \delta$ for all $x$. Let $g \in N\left(S^{1}\right)$ be defined by

$$
g(x)=f(x)+\sum_{i=0}^{n-1} \sigma_{i}(x) \tau_{i}\left(x-f^{i}(q)\right)
$$

where $\tau_{i}=d f\left(f^{i}(p)\right)-d f\left(f^{i}(q)\right)$.

It is easy to see that $g\left(f^{l}(q)\right)=f\left(f^{l}(q)\right)$ for all $0 \leq l \leq n$, and $g$ is $\varepsilon$ close to $f$ in the $C^{1}$ metric, and therefore all periodic points of $g$ are hyperbolic and $\Omega_{c}(g) \subset U_{c}$. These properties imply that $g^{n}(q)=q$ and $q$ is expanding.

Moreover,

$$
\begin{aligned}
d g\left(f^{l}(q)\right) & =d f\left(f^{l}(q)\right)+\sum_{i=0}^{n-1}\left[\sigma_{i}^{\prime}\left(f^{l}(q)\right) \tau_{i}\left(f^{l}(q)-f^{i}(q)\right)+\sigma_{i}\left(f^{l}(q)\right) \tau_{i}\right] \\
& =d f\left(f^{l}(q)\right)+\tau_{l}=d f\left(f^{l}(p)\right)
\end{aligned}
$$

Hence,

$$
\begin{aligned}
d g^{n}(q) & =\prod_{i=0}^{n-1} d g\left(g^{i}(q)\right)=\prod_{i=0}^{n-1} d g\left(f^{i}(q)\right) \\
& =\prod_{i=0}^{n-1} d f\left(f^{i}(p)\right)=d f^{n}(p) .
\end{aligned}
$$

Therefore, $\left|d f^{n}(p)\right|>1$ and the lemma is proved.

Now we shall prove the theorem by adapting a technique due to Mañe [3]. Let $f \in N\left(S^{1}\right)$ be structurally stable. If degree $f=1$ the theorem follows from Peixoto's theorem [6]; so we assume degree $f>1$. By [5, Corollary 2.4], $\Omega(f)=\overline{\operatorname{Per}(f)}$ and, by the proof of Lemma 3.2, $\Omega_{c}(f)$ is finite. Hence to show that $f$ satisfies Axiom A, it is sufficient to show that $\left.f\right|_{\Omega_{e}}(f)$ is expanding, i.e., there exist $k>0$ and $\lambda>1$ such that $\left|d f^{n}(x)\right| \geq k \lambda^{n}$.

By compactness and $f$-invariance of $\Omega_{e}(f)$, this property is equivalent to showing that there exist $n \in \mathbf{N}$ and $c>1$ such that $\left|d f^{n}(x)\right|>c$ for all $x \in \Omega_{e}(f)$. By using the compactness of $\Omega_{e}(f)$ and the chain rule, it is easy to prove that this is equivalent to the fact that for every $x \in \Omega_{e}(f)$ there exists $n=n(x)$ such that $\left|d f^{n}(x)\right|>1$. Hence, everything is reduced to proving this condition.

By contradiction, suppose there exists $x \in \Omega_{e}$ such that $\left|d f^{n}(x)\right| \leq 1$ for all $n \in N$. Then $\left.f\right|_{\omega(x)}$ is not expanding because $\omega(x)$ is compact. Let $S$ be the family of compact $f$-invariant subsets $\Sigma$ of $\omega(x)$ such that $\left.f\right|_{\Sigma}$ is not expanding. It is easy to see that if $\left\{\Sigma_{\alpha} \mid \alpha \in A\right\} \subset S$ satisfies $\Sigma_{\alpha}^{\prime} \subset \Sigma_{\alpha}^{\prime \prime}$ or $\Sigma_{\alpha}^{\prime \prime} \subset \Sigma_{\alpha}^{\prime}$ for all $\alpha^{\prime}, \alpha^{\prime \prime} \in A$ then $\bigcap_{\alpha} \Sigma_{\alpha} \in S$. Hence by Zorn's lemma there exists $\Sigma \in S$ such that $\Sigma^{\prime} \in S$ and $\Sigma^{\prime} \subset \Sigma$ imply $\Sigma^{\prime}=\Sigma$. Since $\left.f\right|_{\Sigma}$ is not expanding there exists $y \in \Sigma$ such that $\left|d f^{n}(y)\right| \leq 1$ for all $n \in N$. Then $\omega(y) \in S$ which, together with the fact that $\omega(y) \subset \Sigma$, implies that $\omega(y)=\Sigma$. It follows that $y$ is a recurrent point of $f$. But by Lemma 3.3, $y$ is not a recurrent point. This contradiction proves the theorem. 


\section{REFERENCES}

1. J. Franks, Necessary conditions for stability of diffeomorphisms, Trans. Amer. Math. Soc. 158 (1971), 301-308.

2. M. V. Jakobson, On smooth mappings of the circle into itself, Math. USSR-Sb. 14 (1971), 163-188.

3. R. Mañe, Contributions to the stability conjecture, Topology 17 (1978), 383-396.

4. Z. Nitecki, Nonsingular endomorphisms of the circle, Global Analysis, Proc. Sympos. Pure Math., Vol. 14, Amer. Math. Soc., Providence, R.I., 1970.

5. __ Factorization of nonsingular circle endomorphisms, Dynamical Systems, Academic Press, New York, 1973.

6. M. Peixoto, Structural stability on two-dimensional manifolds, Topology 1 (1962), 101-120.

Departamento de Matemàtica, Universidade Federal de SÃo Carlos, CeP: 13560, SÃO CARLOS-SP, BRASIL

Current address: Mathematics Institute, University of Warwick, Coventry CV47AL, United Kingdom 